Supporting Information

\title{
Intervalence Charge Transfer of Ruthenium-Nitrogen Moieties Embedded within Nitrogen-Doped Graphene Quantum Dots
}

Limei Chen, ${ }^{\dagger}$ Peiguang Hu, ${ }^{\dagger}$ Christopher P. Deming, ${ }^{\dagger}$ Nan Wang, ${ }^{\dagger}$ Jia En Lu, ${ }^{\dagger}$ and Shaowei Chen $*, \dagger$

† Department of Chemistry and Biochemistry, University of California, 1156 High Street, Santa Cruz, California 95064, United States

$\ddagger$ New Energy Research Institute, School of Environment and Energy, South China University of Technology, Guangzhou Higher Education Mega Centre, Guangzhou 510006, CHINA
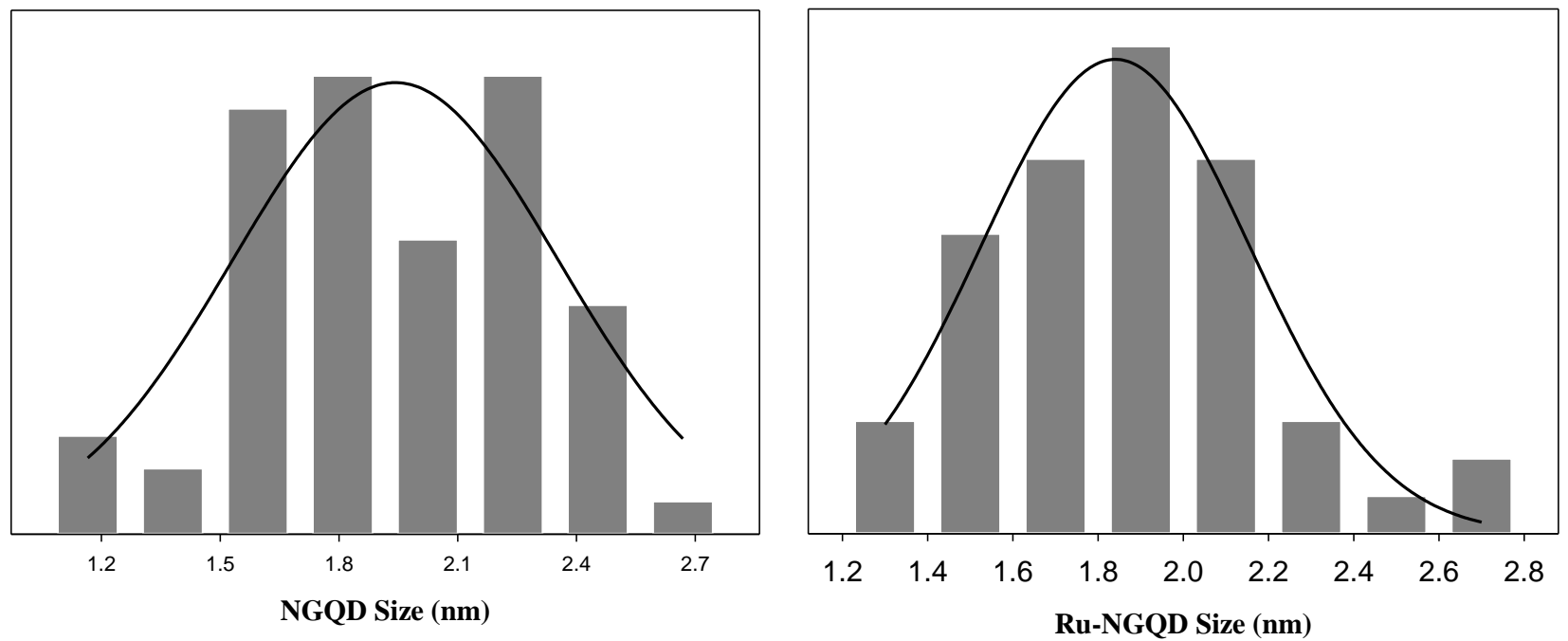

Figure S1. Size histograms of (left) NGQDs and (right) Ru-NGQDs. Data were obtained from Figure 1. Lines are the corresponding Gaussian fits. The average size is estimated to be $2.0 \pm 0.4 \mathrm{~nm}$ and $1.9 \pm 0.3$ $\mathrm{nm}$, respectively. 

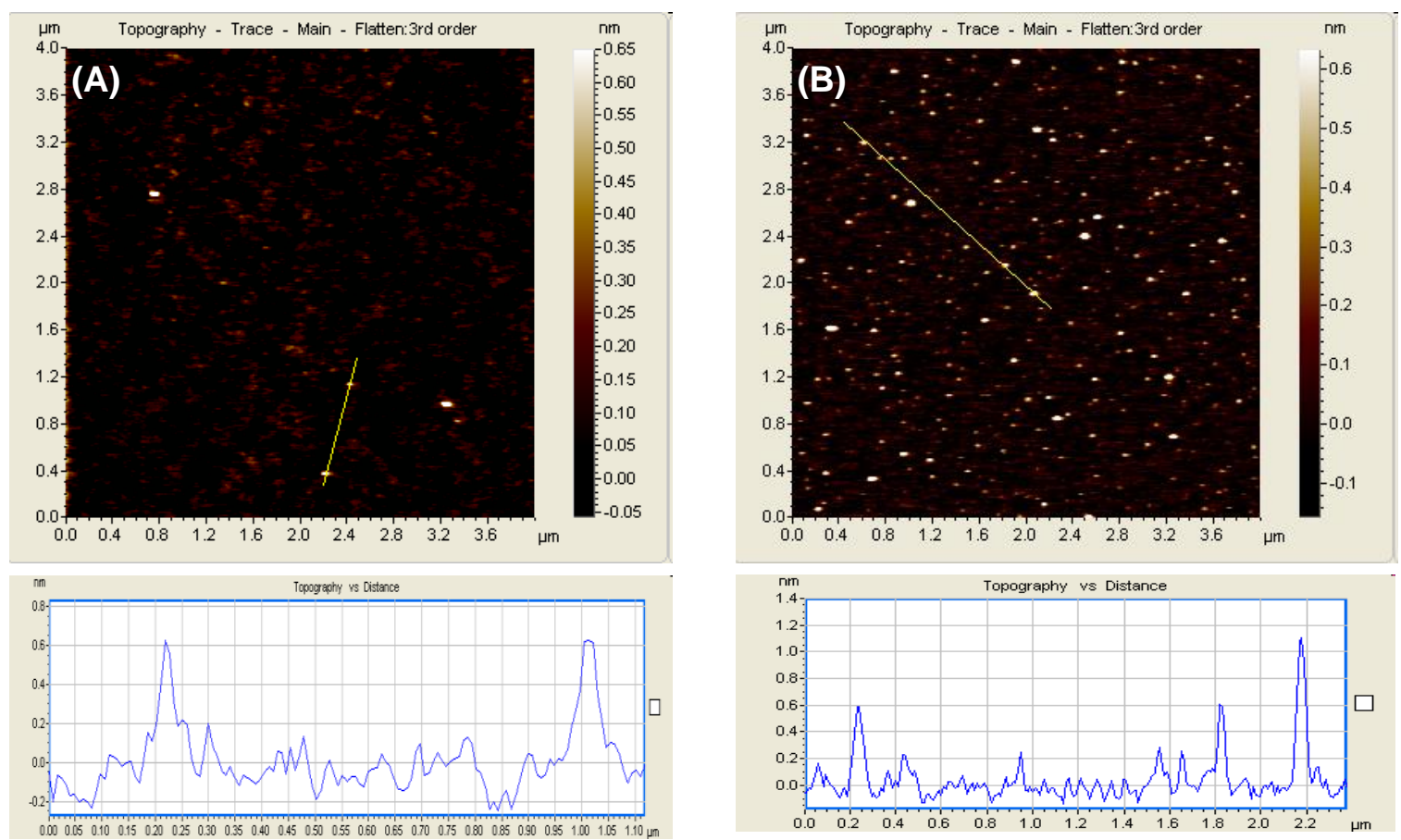

Figure S2. AFM images of (A) NGQDs and (B) Ru-NGQDs deposited on a mica substrate. The bottom panels are the topographic height profiles along the yellow lines in the AFM images.

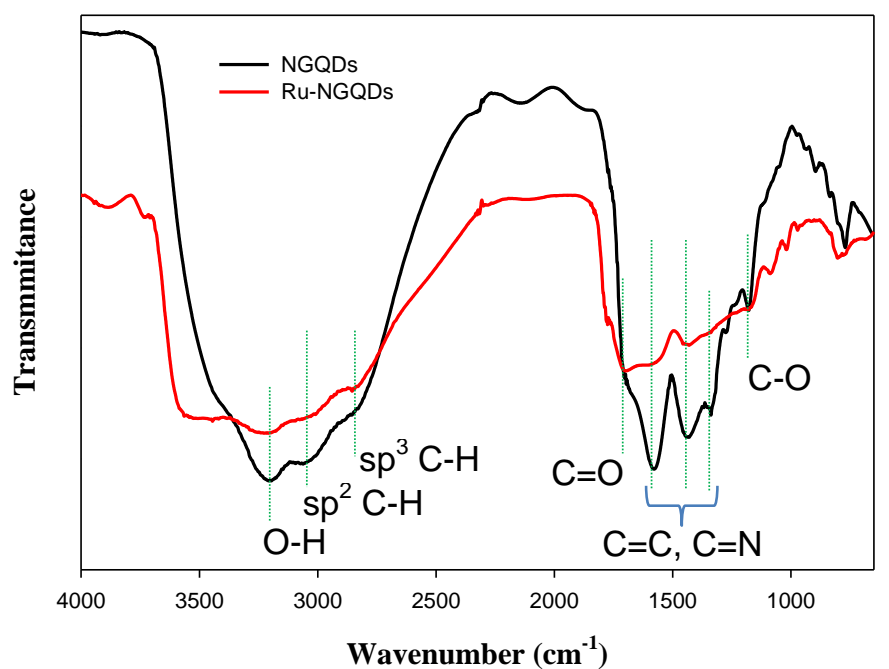

Figure S3. FTIR spectra of NGQDs and Ru-NGQDs. 


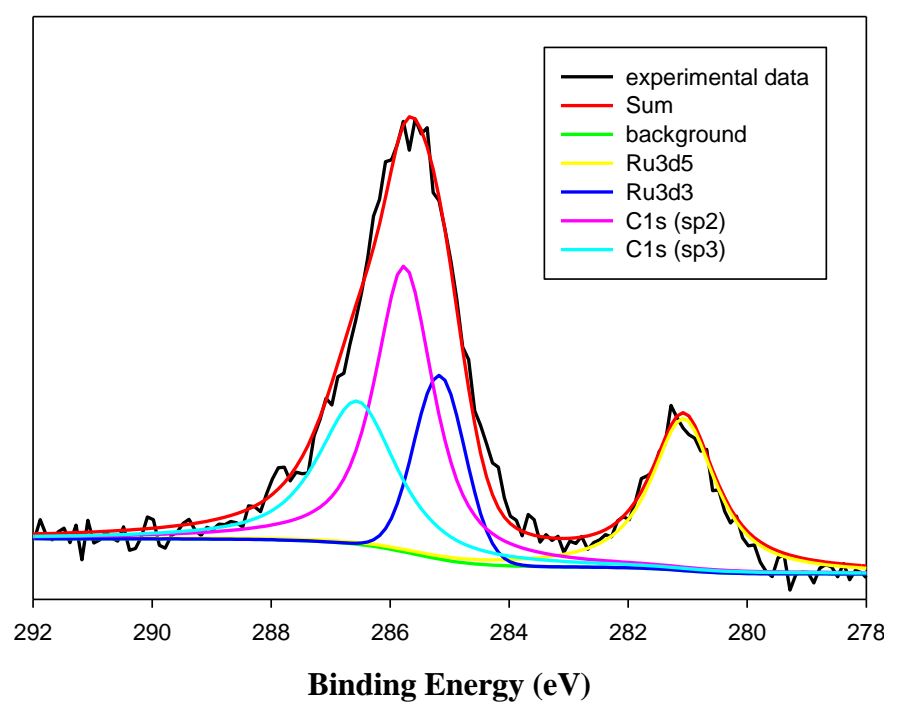

Figure S4. XPS spectrum of the $\mathrm{C} 1 \mathrm{~s}$ and Ru3d electrons of $\mathrm{RuCl}_{2}(\mathrm{Py})_{4}$ synthesized from $\mathrm{RuCl}_{2}(\mathrm{DMSO})_{4}$. Black curve is the experimental data and colored curves are deconvolution fits. The Ru3d electrons are identified at 281.1 and $285.2 \mathrm{eV}$. $\mathrm{RuCl}_{2}(\mathrm{Py})_{4}$ was synthesized by following a literature protocol (I.P.Evans, A. Spencer and G.Wilkinson, Dichlorotetrakis(dimethyl sulphoxide)ruthenium(II) and its use as a source material for some new ruthenium(II) complexes. J. Chem. Soc., Dalton Trans., 1973, 204-209). Experimentally, $30 \mathrm{mg}$ of $\mathrm{RuCl}_{2}(\mathrm{DMSO})_{4}$ was added into $5 \mathrm{~mL}$ of neat pyridine and refluxed at $119{ }^{\circ} \mathrm{C}$ for $30 \mathrm{~min}$. The volume was then reduced and the complex was recrystallized and purified with ethanol.
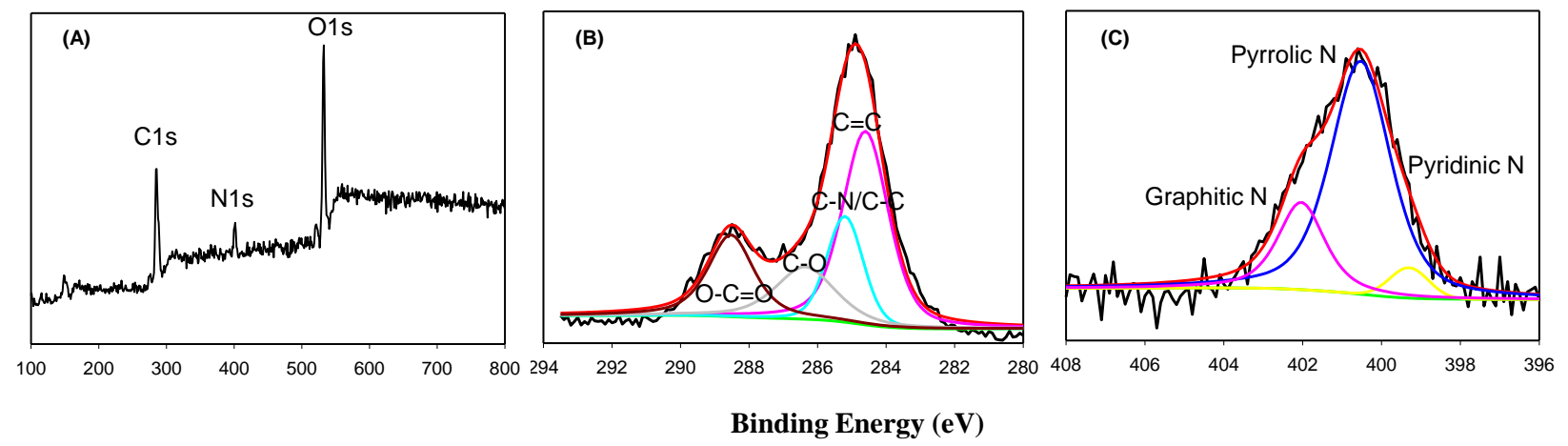

Figure S5. (A) XPS survey spectrum of NGQDs and the corresponding high-resolution scans of (B) C1s and $(C)$ N1s electrons. Black curves are experimental data and colored curves are deconvolution fits. 


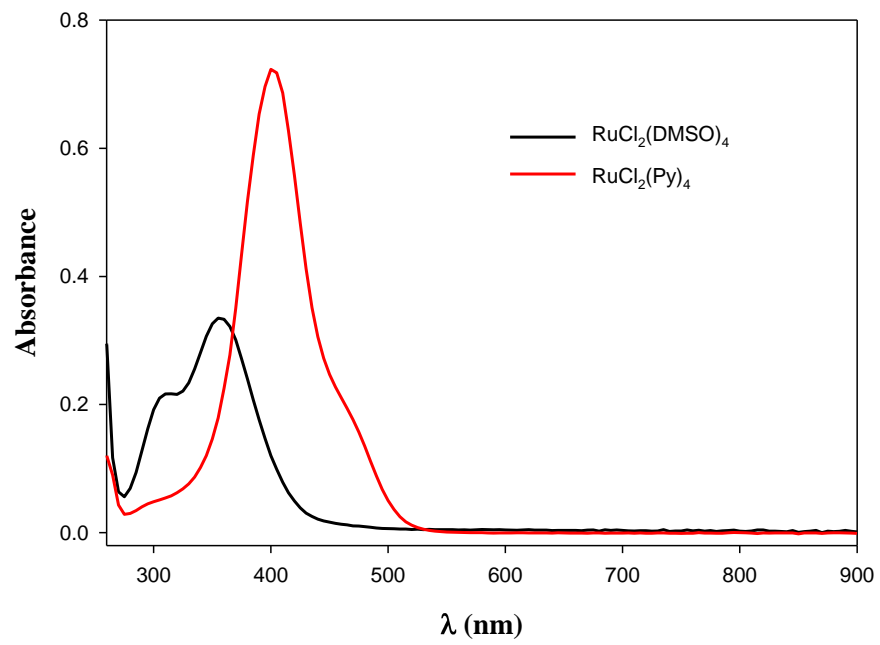

Figure S6. UV-vis spectra of $\mathrm{RuCl}_{2}(\mathrm{DMSO})_{4}$ and $\mathrm{RuCl}_{2}(\mathrm{Py})_{4}$ in DMF.

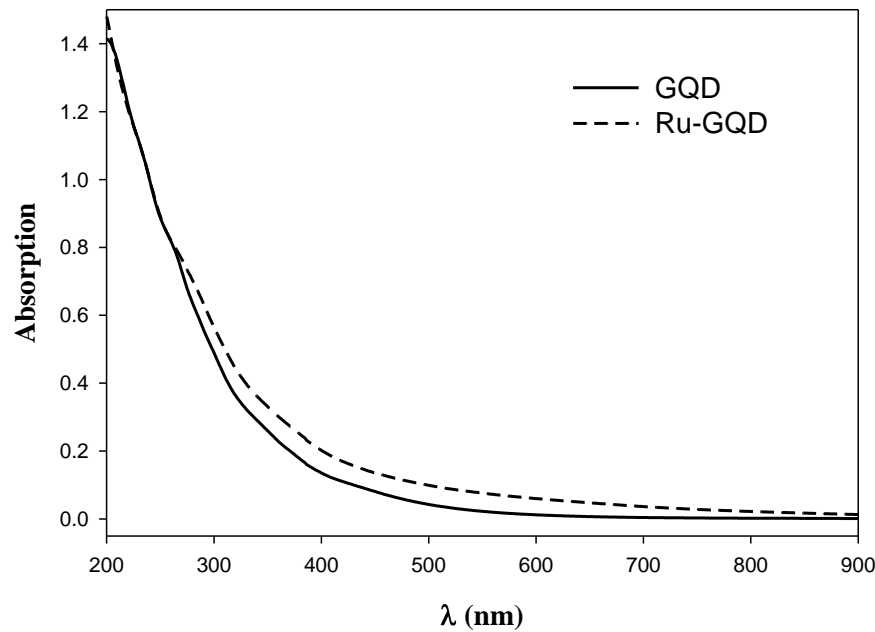

Figure S7. UV-vis spectra of undoped GQDs before and after mixing with ruthenium ions in water. The spectra are virtually invariant, suggesting the lack of complexation of ruthenium ions to the GQDs. 


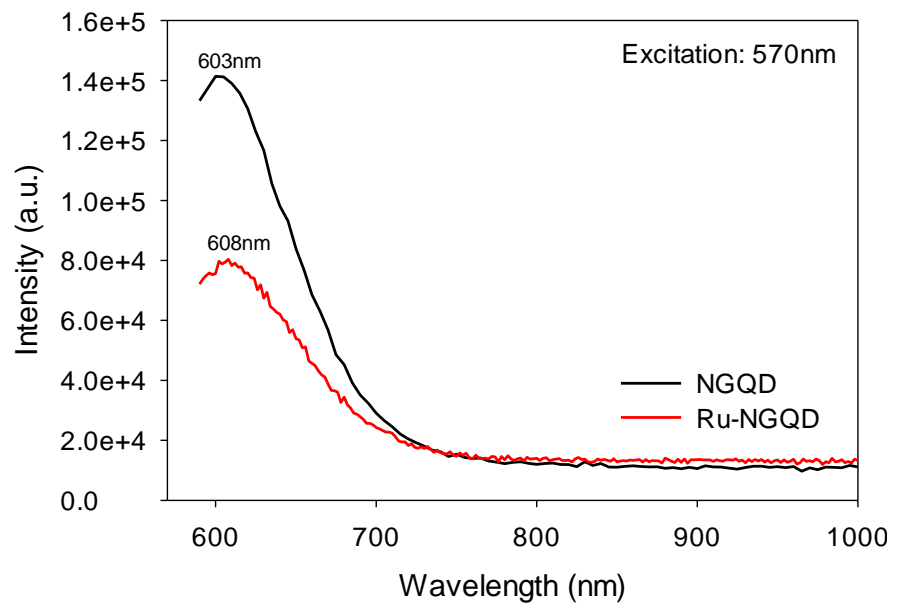

Figure S8. Photoluminescence emission spectra of NQGD and Ru-NGQD in water at the excitation wavelength of $570 \mathrm{~nm}$. Experimental conditions are the same as those in Figure 4. NGQDs exhibited an emission peak at $603 \mathrm{~nm}$, which red-shifted slightly to $608 \mathrm{~nm}$ with Ru-NGQDs. The emission intensity diminished by about $50 \%$ from NGQDs to Ru-NGQDs. 